\title{
Estimation of the exposure for the air shower detection mode of EUSO-SPB1
}

\author{
K. Shinozaki*, M. Bertaina, F. Bisconti $\dagger$ F. Fenu, S. Ferrarese, S. Monte ${ }^{\ddagger}$ \\ University of Turin and/or INFN Torino, Turin, Italy \\ E-mail: kenji.shinozakidto.infn.it
}

\section{A. Anzalone, A. Bruno}

INAF-IASF Parlermo, Palermo, Italy

\section{S. Briz}

University Carlos III de Madrid, Madrid, Spain

A. Diaz

IRAP, University of Toulouse, Toulouse, France

\section{J. Eser, L. Wiencke}

Colorad School of Mines, Golden, USA

\section{A. Olinto}

University of Chicago, Chicago, USA

\section{Vrabel}

Technical University of Kosice, Kosice, Slovakia

\section{for the JEM-EUSO collaboration ${ }^{\S}$}

EUSO-SPB1 was a balloon-borne pathfinder mission of the JEM-EUSO (Joint Experiment Missions for the Extreme Universe Space Observatory) program. A 12-day long flight started from New Zealand on April 25 th, 2017 on-board the NASA's Super Pressure Balloon. With capability of detecting EeV energy air showers, the data acquisition was performed using a $1 \mathrm{~m}^{2}$ two-Fresnel-lens UV-sensitive telescope with fast readout electronics in the air shower detection mode over $\sim 30$ hours at $\sim 16-30 \mathrm{~km}$ above South Pacific. Using a variety of approaches, we searched for air shower events. Up to now, no air shower events have been identified. The effective exposure, regarding the role of the clouds in particular, was estimated based on the air shower and detector simulations together with a numerical weather forecast model. Compared with the case assuming the fully clear atmosphere conditions, more than $\sim 60 \%$ of showers are detectable regardless the presence of the clouds. The studies in the present work will be applied in the follow-up pathfinders and in the future full-scale missions in the JEM-EUSO program.

36th International Cosmic Ray Conference -ICRC2019-

July 24th - August 1st, 2019

Madison, WI, U.S.A. 


\section{Introduction}

The origin of the ultra-high energy cosmic rays (UHECRs; referred to as $\sim$ EeV energies and higher) is still an open question in the today's astrophysics [四]. In particular, above $\sim 100 \mathrm{EeV}$, the UHECR fluxes are on the an order of 1 per $\mathrm{km}^{2}$ in a millennium or even fewer with a steepening spectral index which is interpreted as the well-known Greisen-Zatsepin-Kuz'min mechanism during the propagation of UHECRs or/and acceleration limit of UHECRs at their sources. To further investigate the nature of UHECRs, larger exposures are essential.

The JEM-EUSO (Joint Experiment Missions for Extreme Universe Space Observatory) program [] aims at space-based UHECR observations from satellite orbits. Using an ultra-wide field-of-view (FOV) UV telescope, the fluorescence technique enhances instantaneous apertures to efficiently increase the exposure. In our framework, two full-scale missions are promoted as K-EUSO (KLYPVE-EUSO) [3] and POEMMA (Probe Of Extreme Multi-Messenger Astrophysics) [四]. The former will be based on the International Space Station (ISS). To test the key technologies developed for JEM-EUSO, we had conducted pathfinders, a total of 130 hours of oper-

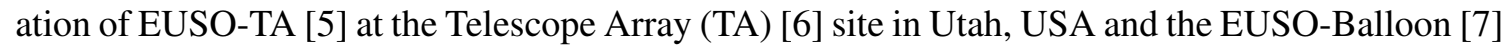
on the one night stratospheric CNES (French Space Agency) balloon flight in Timmins, Canada.

In space-based UHECRs observations, several factors are needed to be taken into account [ []]. In particular, the trigger conditions should be optimized to fit the telemetry limit. Air shower signals originate in the atmosphere behind the airglow background light that is persistently emitted in the mesosphere altitude around $90 \mathrm{~km}$ above sea level. The intensity of such diffuse light determines threshold energy of detectable UHECR events. The presence of the clouds in the FOV partly reduced the instantaneous apertures in terms of area and solid angle acceptance.

In April 2017, we conducted the EUSO-SPB1 mission to operate the first fluorescence telescope flown to sub-orbital space with the capability of air shower detection. In the present work, we evaluated the effective exposure for EUSO-SPB1 mainly focusing on the role of the clouds. We will remark the relevant issues in the future space-based UHECR observations.

\section{EUSO-SPB1 mission}

The EUSO-SPB1 telescope [Q] consisted of a $1 \mathrm{~m}^{2}$ aperture two-Fresnel-lens optics and a photo-detector module (PDM) [ए]] composed of 36 multi-anode photomultiplier tubes (MAPMTs) covered with BG3 UV-band-pass filters. The total of 2304 pixels cover an $\sim 11^{\circ}$ FOV with $\sim 0.2^{\circ}$ spatial pitch and small non-efficient parts among MAPMTs. In all the pixels, photon counting is performed every $2.5 \mu \mathrm{s}$. This time duration is called a gate time unit (GTU).

Compared with other JEM-EUSO pathfinders, the major characteristics of EUSO-SPB1 was an autonomous trigger to detect air showers in the EeV regime [W]. Once a trigger is issued, the counts on all the pixels were saved for 128 GTUs. Prior to the flight, a field test of EUSO-SPB1 was carried out at the TA site [目] to verify and to quantify the trigger and other key functions using

\footnotetext{
* Speaker.

${ }^{\dagger}$ Affiliated to INFN

$\ddagger$ Affiliated to University of Turin

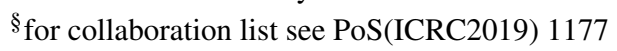




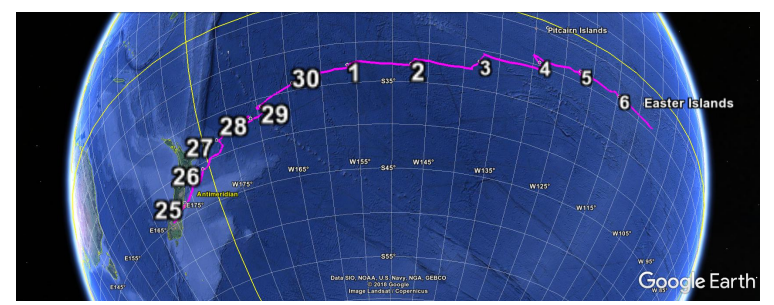

Figure 1: EUSO-SPB1 flight track. The positions at 12:00 UTC are labeled by the date.

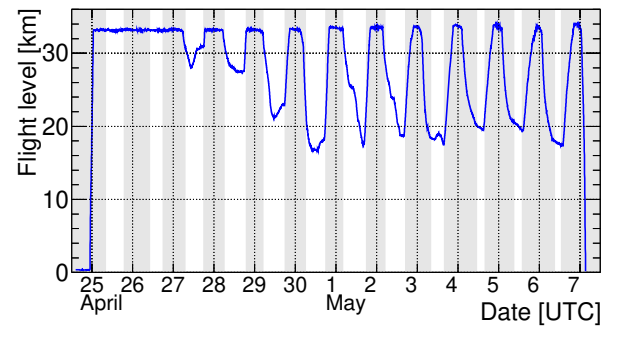

Figure 2: EUSO-SPB1 flight level. Bright regions indicate hours with any DAQ made.

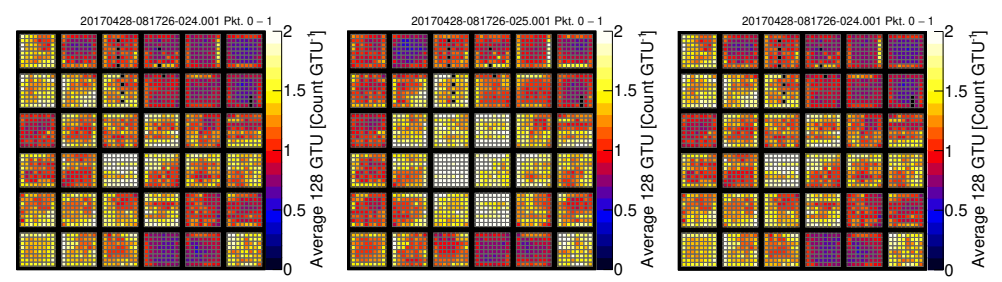

Figure 3: Example of the background images on the FOV measured by the EUSO-SPB1 telescope. These images are the averaged 10 adjacent triggers. Each panel corresponds to $\sim 1$ min time interval.

a movable laser device and the TA's Central Laser Facility. By detecting signals from the scattered light of laser shots, we evaluated the performance of EUSO-SPB1 [Q].

Figure $\square$ shows the EUSO-SPB1 track on the Google Earth image. Positions as of 12:00 UTC are labeled with the day of the month. EUSO-SPB1 resided around the Anti-meridian. This time corresponding to around the local midnight (hereafter nights mean the local nights of UTC dates).

Figure $\square$ displays the variation of EUSO-SPB1 flight level. Bright regions indicate hours in which any data acquisition (DAQ) was made by the EUSO-SPB1 telescope.

EUSO-SPB1 onboard NASA's Super Pressure Balloon (SPB) was released from the Wanaka airport, New Zealand on April 24th, 2017 at 22:50 UTC, around the noon of April 25th local time. EUSO-SPB1 first reached the nominal flight level of $\sim 33 \mathrm{~km}$ in 2 hours. Since April 27th, the flight level tended to drop due to the unexpected gas leakage on the balloon envelope. While the efforts for recovering EUSO-SPB1 on the continent, the flight level finally became $\sim 17 \mathrm{~km}$. The flight lasted $\sim 12.4$ days over $\sim 7000 \mathrm{~km}$ until we had to abandon the payload off Easter Island.

Since 08:31 UTC of April 25th, we operated the air shower detection mode every night for $\sim 30$ hours in total. The operable time decreased night by night due to the waxing Moon in the sky. In the end, a $\sim 27$ hours of the data, in part reduced to a 25 GTU length were transmitted to the ground before EUSO-SPB1 sunk. Excluding commissioning phase, $~ 21.7$ hours of the data were acquired when all MAMPTs were operational. The EUSO-SPB1 instrument had been operational till the end. We confirmed the functionality of the trigger from trigger rates and background levels [W]. Using a variety of approaches from visual inspection to machine learning, we search the triggered events for air shower event. Up to now, no candidates have been found [12], [3]].

Figure [3] shows selected images of background in the FOV of the EUSO-SPB1 telescope. These images are averaged over the 10 adjacent triggers, a time span of $3.2 \mathrm{~ms}$. Each panel corresponds to $\sim 1$ min time interval [14]]. This figure is also intended to illustrate the deployment of 

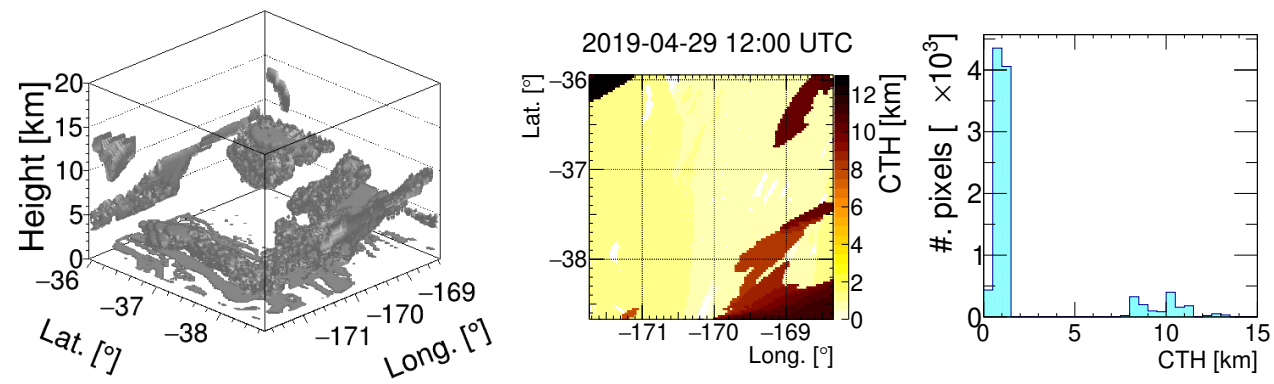

Figure 4: A WRF output for 12:00 UTC of April 29th. Left: visualization of grid-boxes with a non-zero cloud fraction. Middle: CTH map around the EUSO-SPB1 position. Right: CTH distribution in this area.

MAMPTs in the PDM. The color scales denote the background level in units of counts per GTU.

In these images, the cloud pattern and its relative motion with EUSO-SPB1 was also recognized. Over its flight, EUSO-SPB1 oversaw various weather situations. To monitor the presence of clouds in the FOV, an infrared camera was operated in limited times along with the EUSO-SBP1 telescope and also in the daytime. Preliminary results based on a possible method [ㄷ]] that could be applied to the data are presented in Reference [16].

\section{Cloud-top height estimations and air shower simulations}

The role of clouds is important factor to the performance of the air shower detections for EUSO-SPB 1 and for future pathfinders and full-scale missions. In the present work, we utilized the WRF (Weather Research and Forecasting) model [ㄱ] to estimate the cloud-top height (CTH). WRF is a mesoscale meteorological model developed by the National Center for Atmospheric Research. It can reach up to a $1 \mathrm{~km}$ horizontal spatial resolution. WRF provides the atmospheric quantities for each pixel over all vertical levels on the model grid. Further details for this specific study is given in Reference [1[8]. The key result from WRF was presented in Reference [144]. Regional weather forecasting models in general simulate the atmospheric quantities staring with an input of a global circulation model. We employed ECMWF (European Centre for Medium-range Weather Forecasts) data [19] for such inputs. CTH retrieval was based on the cloud fraction which expresses the cloudiness in a model grid-box. Outputs are given in 10-minute intervals for gridboxes of a $\sim 3 \mathrm{~km}$ pixel over 49 pressure levels below $\sim 30 \mathrm{~km}$ height. We defined CTH by the highest grid-box with a cloud fraction greater than 0.2 on horizontal pixel.

Figure $\$$ displays a WRF output for 12:00 UTC of April 29th. The left panel visualizes gridboxes with a non-zero cloud fraction seen from southwest. The middle and right show the CTH map and distribution in a $\sim 300 \mathrm{~km} \times 300 \mathrm{~km}$ area around the EUSO-SPB1 position, respectively.

Figure 5 displays the CTH variation from April 24th through May 2nd. Bright regions denote the night time between twilights at the EUSO-SPB1 position.

The WRF results show that the CTH variation was moderately small and was as low as a few kilometers until April 29th. In the following nights, CTH reach the height of tropopause. The CTH variation are used for studying the cloud impact on the air shower detection with the simulations. 


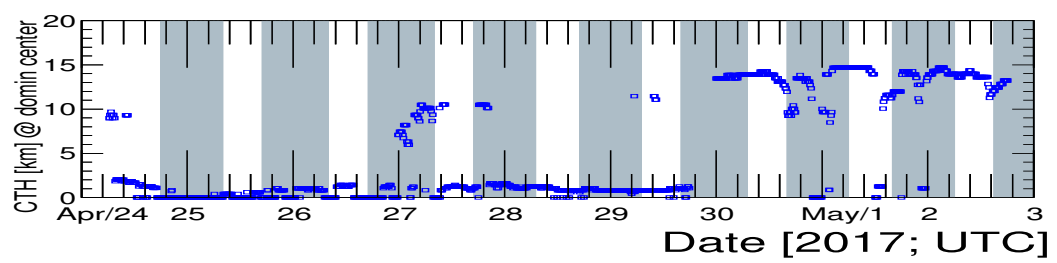

Figure 5: CTH variation between April 24th and May 2nd. Bright regions are night time at EUSO-SPB1.

So far, we have estimated how many air shower events could be detected during the EUSOSPB1 flight. Using the air shower and detector simulations with 'EUSO OffLine' [20], it turned out to be $1.2 \pm 0.4$ event for $21.7 \mathrm{~h}$ assuming the clear atmospheres [Q]. These simulations take into account the calibration of the EUSO-SPB1 telescope in the laboratory. The peak energy of the triggered events has been found consistent with the laser detection results in the field test.

In the present work, we investigate the role of the cloud in the air shower detection using the ESAF (EUSO Simulation and Analysis Framework) software [2]]. For the air shower detection from the above, CTH and the optical depth of the cloud are key factors for the detection efficiency. For a quantification of the cloud impact during the EUSO-SPB1 flight, we assumed the CTH variation as shown in Figure [1. In the former work [ [8], we defined the parameter called 'cloud efficiency' $\kappa_{\mathrm{C}}$ as the ratio in apertures of air shower detections, namely equivalent to the ratio in event rates estimated by assuming the modeled cloudy conditions to the those estimated under the clear atmosphere conditions.

To determine this value for EUSO-SPB1, we first simulated the air showers with the case there is no clouds in the FOV by assuming a omni-directional isotropic UHECR flux given in Reference [[2]]. The background level was taken from the average of the real data [W], [4]]. The event rates for three typical flight levels of $17 \mathrm{~km}, 21 \mathrm{~km}$, and $28 \mathrm{~km}$ were estimated. The overall rate was estimated by weighting the durations of DAQ in different levels. To correct uncertainty in the energy scale due to the models used in ESAF and OffLine, we re-scaled to fit the above mentioned event rate obtained by OffLine.

Figure $\mathbf{6}$ displays fluorescence photon density at a EUSO-SPB1 level of $28 \mathrm{~km}$ as a function of the originating height. In the clear atmospheres, air showers induced by a $3 \mathrm{EeV}$ proton towards the sub-balloon point on sea level. Different incident zenith angles $\vartheta=0^{\circ}, 45^{\circ}, 60^{\circ}$, and $75^{\circ}$, were simulated. The effect of Rayleigh scattering by molecules in the air and absorption by ozone is included. This figure is intended to illustrate from which height how many photons reach the EUSO-SPB1 telescope regardless the FOV covered by the PDM.

Within a limited energy range, the heights where air showers develop depend on the incident zenith angles, i.e., the larger zenith angles, air showers develop the closer to the observation level. On the other hand, as seen for the case of $\vartheta=75^{\circ}$, the location of the shower development can displace far away from the FOV. For a lower flight level of $17 \mathrm{~km}$ for example, the characteristics of this figure are modified by other factors. Air showers may develop even a few kilometers away and lower density of the air allows more photons to reach EUSO-SPB1. The vertical depth corresponds to $\sim 90 \mathrm{~g} \mathrm{~cm}^{-2}$. The mean free path of UHECRs in EeV energies is $\sim 45-60 \mathrm{~g} \mathrm{~cm}^{-2}$ in the air [231, 24]]. Thus, most of UHECRs initiate air showers above this level.

Figure $\square$ display examples of simulated air shower signals on the PDM with the same condi- 


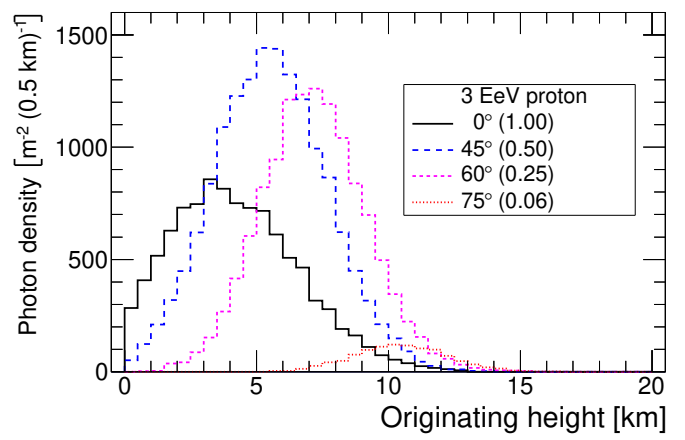

Figure 6: Fluorescence photon density at a $28 \mathrm{~km}$ height as a function of the originating height. Air showers from a $3 \mathrm{EeV}$ proton were simulated towards the sub-balloon point.

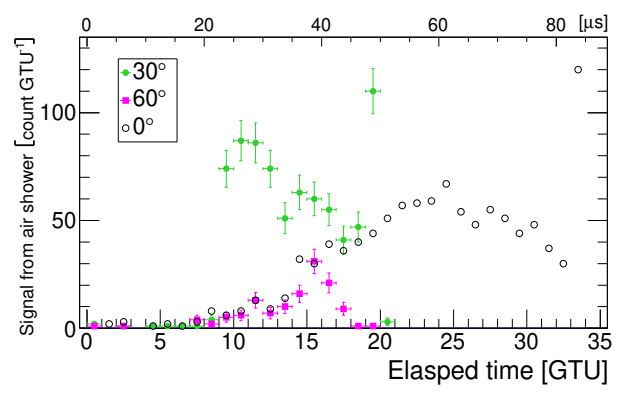

Figure 7: Examples of simulated air shower signals on the PDM with the same conditions as in Figure $\mathbf{B}$ but for three different zenith angles. The horizontal axis is elapsed time since the first signal detection.

tions as in Figure 6 but for zenith angles of $30^{\circ}$ and $60^{\circ}$ cases, and $0^{\circ}$ case for comparison.

An unlikely $\vartheta=0^{\circ}$ example represents a case that the whole shower development takes place within the FOV. The largest excess is a result of Cherenkov light back-scattered from the sea. In this case, stationary but variable signals are seen on the PDM. In other cases, a light spot moves on the background. The time profile of the detected signals largely depends on which part of showers is observable within a limited FOV of EUSO-SPB1. Among these examples, only the $60^{\circ}$ case does not trigger. Typically, large zenith angles are more advantageous if the maximum of the shower development takes place near or in the FOV,

In the same way, simulating air showers in the cloudy conditions were made by assuming one-layer cloud over the whole FOV at the sampled CTH from WRF outputs. We conservatively assumed the optical depth of cloud to be 1 . Thus, all the photons originate below the CTH are attenuated by $\exp (-1)$ before reaching EUSO-SPB1 in addition to the effect suffered from the clear atmospheres. If 'not very old' air showers reach the cloud level in the FOV, more intense back-scattered Cherenkov light may be seen compared with those from sea as is seen in Figure $\square$.

Figure $\$$ displays the cloud efficiency as a function of the primary energy. Error bars denote the uncertainty due to simulated statistics with the UHECR spectrum.

For this spectrum, the overall $\langle\kappa\rangle$ value is $\sim 62 \%$ above $1 \mathrm{EeV}$. We used a conservative threshold of the cloud fraction of 0.2 from the WRF outputs that tends to give a CTH above the height that effectively matters to the photon propagation from the air showers. One-layer optically thick model clouds attenuate more than the usual case. The average background level that includes the contribution from the clouds is also used in simulations for the clear atmospheres. These assumed cloud properties allow for setting a lower limit on the estimated number of detectable air shower events for EUSO-SPB1.

Figure $\mathrm{Q}$ displays the occurrence of the CTH from the WRF output during the DAQ in the air shower detection mode. The maximum height of shower developments are compared by assuming the typical $X_{\max }$ values of $\sim 780 \mathrm{~g} \mathrm{~cm}^{-2}$ and $\sim 680 \mathrm{~g} \mathrm{~cm}^{-2}$ for $\sim 3 \mathrm{EeV}$ proton and iron UHECRs, respectively from Reference [D25]. A $\sim 100 \mathrm{EeV}$ proton case of $X_{\max }=860 \mathrm{~g} \mathrm{~cm}^{-2}$ that is relevant for the full-scale missions is also shown. 


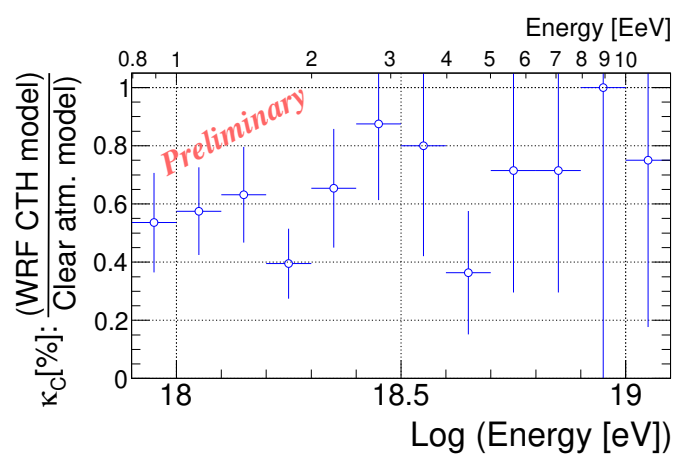

Figure 8: Cloud efficiency as a function of the energy. Error bars denote the uncertainty due to simulated statistics with the UHECR spectrum.

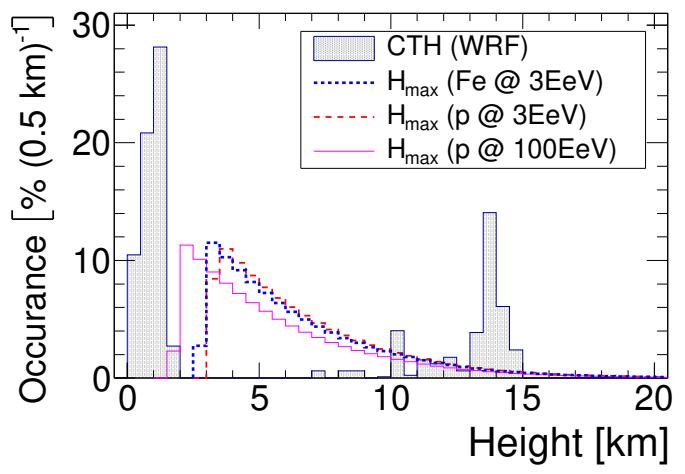

Figure 9: CTH distribution from the WRF output during DAQ compared with those of the maximum height of air shower developments.

Two distinct populations are found. One is below $1.5 \mathrm{~km}$ and the other is above $7 \mathrm{~km}$. The lower $\mathrm{CTH}$ population accounts for the $\sim 60 \%$ occurrence. In such situations, a substantial portion of fluorescence photons from the air showers originate above CTH is compared in Figure 6 and are not affected towards EUSO-SPB1. The higher CTH population practically prevents the photons in the other way round. The $\langle\kappa\rangle$ value of $\sim 60 \%$ is naturally explained by this population and does not seem to depend much on primary particles in the $\mathrm{EeV}$ energies.

\section{Summary and outlook}

From the present work, more than $\sim 60 \%$ of the air showers in the EeV regime that would trigger EUSO-SPB1 in clear atmosphere conditions also trigger even in the cloudy conditions based on the WRF output. The expected number of the detected air showers during the DAQ time of EUSO-SPB1 is still on the order of one or fewer. Considering SPB's $\sim 100$ day long flight potential, high probability of detecting shower events was expected if flight could have been longer. Now, the collaboration is in preparation of the 'EUSO-SPB2' mission anticipating launch in 2022.

In the full-scale space-based missions like K-EUSO and POEMMA, more than 50 PDMs will be deployed to cover even wider FOV. They allow for measurement of the entire picture of the shower development above a few times $10 \mathrm{EeV}$ energies. The science cases require not only high statistics of UHECR observations but also determination of exposures throughout continuously varying conditions. EUSO-SPB1 was the first platform with the autonomous trigger to study such situations. With a $25 \mathrm{~cm}$ telescope onboard the ISS, the Mini-EUSO mission will be carried out in 2019 mainly aimed at measurement of the background. Experience, knowledge, and developed methods gained through the pathfinder missions will be applied in the future missions.

\section{Acknowledgments}

This work was partially supported by NASA grants NNX13AH54G, NNX13AH52G, French Space Agency (CNES), Italian Space Agency through the ASI INFN agreement n. 2017-8-H.0, Italian Ministry of Foreign Affairs and International Cooperation, the Basic Science Interdisciplinary Research Projects of RIKEN and JSPS KAKENHI Grant (22340063, 23340081, and 24244042), 
Deutsches Zentrum für Luft und Raumfahrt, and 'Helmholtz Alliance for Astroparticle Physics HAP' funded by the Initiative and Networking Fund of Helmholtz Association (Germany). We acknowledge NASA Balloon Program Office, Columbia Scientific Balloon Facility, Telescope Array Collaboration, and Wanaka airport. The present research used resources of the National Energy Research Scientific Computing Center (NERSC), a US Department of Energy Office of Science User Facility operated under Contract No. DE-AC02-05CH11231.

\section{References}

[1] B.R. Dawson, M. Fukushima, and P. Sokolsky, Prog. Theor. Exp. Phys., 12 (2017) 12 A107.

[2] M. Bertaina, Proc. 36th Int. Cosmic Ray Conf. (Madison), Pos (ICRC2019) 192 (2019).

[3] M. Casolino et al., Proc. 35th Int. Cosmic Ray Conf. (Busan), Pos (ICRC2017) 368 (2019).

[4] A.V. Olinto et al., Proc. 36th Int. Cosmic Ray Conf. (Madison), Pos (ICRC2019) 378 (2019).

[5] F. Bisconti et al., Proc. 36th Int. Cosmic Ray Conf. (Madison), Pos (ICRC2019) 197 (2019).

[6] H. Tokuno et al., Nucl. Instrum. Meth. A, 76 (2013) 54.

[7] G. Abedellaoui et al., J. Astropart. Phys. 111 (2019) 54.

[8] J.H. Adams Jr. et al., Astropart. Phys. 44 (2013) 76.

[9] J. Eser et al., Proc. 36th Int. Cosmic Ray Conf. (Madison), Pos (ICRC2019) 247 (2019).

[10] J.H. Adams et al., Exp. Astron. 40 (2015) 19.

[11] M. Battisti et al., Nucl. Instrum. Meth. A936 (2019) 349.

[12] A. Diaz et al., Proc. 36th Int. Cosmic Ray Conf. (Madison), Pos (ICRC2019) 240 (2019).

[13] M. Vrabel et al., Proc. 36th Int. Cosmic Ray Conf. (Madison), Pos (ICRC2019) 456 (2019).

[14] K. Shinozaki et al., EPJ Web of Conferences 210 (2010) 05006

[15] A. Anzalone et al., IEEE Trans. Geosci. Remote Sens. 57 (2019), 304

[16] A. Bruno et al., Proc. 36th Int. Cosmic Ray Conf. (Madison), Pos (ICRC2019) 454 (2019).

[17] National Center for Atmospheric Research (NCAR), 'WRF the Weather Research and Forecasting Model', http://www.mmm.ucar.edu/weather-research-and-forecasting-model

[18] S. Monte et al., in these proceedings.

[19] ECMWF I Advancing global NWP through international collaboration, https://www.ecmwf.int/

[20] T. Paul et al., Proc. 35th Int. Cosmic Ray Conf., Pos (ICRC2017) 443.

[21] C. Berat et al. Astropart. Phys., 33 (2010) 211.

[22] F. Fenu, Proc. 35th Int. Cosmic Ray Conf., Pos (ICRC2017) 486.

[23] R.U. Abbassi et al., Phys. Rev. D 92 (2015) 032007.

[24] P. Abreu et al., Phys. Rev. Lett. 109 (2012) 062002.

[25] J. Bellido et al., Proc. 35th Int. Cosmic Ray Conf., Pos (ICRC2017) 506.

[26] F. Capel et al., Adv. Space Res. 62 (2018) 2954. 